Acta Crystallographica Section D

Biological

Crystallography

ISSN 0907-4449

\section{Biochemical, biophysical and preliminary X-ray crystallographic analyses of the fusion core of Sendai virus $F$ protein}

It is emerging that enveloped viruses may adopt a unique entry/fusion mechanism; in paramyxoviruses, including Sendai virus $(\mathrm{SeV})$, the attachment protein $\mathrm{HN}$ (or its homologue $\mathrm{H}$ or $\mathrm{G}$ ) binds a cellular receptor which triggers conformational changes of its fusion protein, $\mathrm{F}$. There are at least three conformations of the $\mathrm{F}$ protein in the current fusion model: the pre-fusion native conformation, the prehairpin intermediate conformation and the post-fusion coiled-coil conformation. The fusion mechanism of $\mathrm{SeV}$, a member of the Paramyxoviridae family, has been well established and several structural and functional domains or modules have been proposed from studies of its $\mathrm{F}$ protein. However, biochemical and biophysical studies of the heptad-repeat (HR) regions (HR1 and HR2) have not been systematically carried out. HR1 and HR2 strongly interact with each other to form a stable six-helix coiled-coil bundle as the postfusion conformation. In this study, a single-chain HR1-linker-HR2 protein of $\mathrm{SeV}$ was prepared in an Escherichia coli expression system and biochemical and biophysical analyses showed it to form a typical six-helix coiled-coil bundle; its trigonal crystals diffracted X-rays to $2.5 \AA$ resolution. The crystal structure will help to reveal the structural requirements of the post-fusion coiled-coil conformation of SeV F protein.
Received 23 April 2004 Accepted 29 June 2004
Correspondence e-mail: vetdean@cau.edu.cn george.gao@ndm.ox.ac.uk
(C) 2004 International Union of Crystallography Printed in Denmark - all rights reserved

\section{Introduction}

Sendai virus $(\mathrm{SeV})$ is the prototype of genus Respirovirus of the Paramyxoviridae family, which includes five previously defined and two newly designated genera (the genera Rubulavirus, Respirovirus, Morbillivirus, Pneumovirus, Metapnuemovirus and the new Avulavirus and Henipavirus; Lamb et al., 2000; Wang et al., 2001; Chang et al., 2001). SeV is also called murine parainfluenza virus 1 and has been extensively studied as a model system for paramyxovirus infection, especially in virus-lipid-membrane fusion (reviewed in Peisajovich \& Shai, 2002, 2003). The Paramyxoviridae family is a group of enveloped negative-stranded RNA viruses that infect a broad spectrum of species, including humans, domestic (farm and pet) and wild animals (Lamb et al., 2000). Entry of paramyxovirus into the host cells is the first step in virus infection and is mediated by viral and cellular membrane fusion (Lamb, 1993; Lamb et al., 1999; Peisajovich \& Shai, 2002, 2003; Morrison, 2003). During this process, the viral envelope proteins embedded on the virion surface play an important role. In $\mathrm{SeV}$, there are two envelope proteins on the surface: haemagglutinin neuraminidase $(\mathrm{HN})$ and fusion protein (F). HN, called the attachment protein, is responsible for initial binding to the sialic acid- containing target-cell receptor and the process triggers conformational changes in the $F$ protein (reviewed in Peisajovich \& Shai, 2002, 2003). F protein is initially synthesized as an inactive precursor $F_{0}$ and subsequently cleaved by a cellular furin-like enzyme into two disulfide-bond-linked subunits, $F_{1}$ and $F_{2}$. The covalently linked $F_{1}$ and $F_{2}$ form a trimer on the virion surface (Lamb, 1993). There are several important functional or structural modules/domains in the $\mathrm{SeV} F$ protein, some of which are confirmed to be common to all paramyxoviruses. These include the highly conserved heptad-repeat (HR) regions, $\mathrm{F}_{1}$ $\mathrm{N}$-terminal fusion peptide (FP), internal fusion peptide (IFP) and an additional internal leucine-zipper (LZ) domain with a virus-fusion inhibition effect (reviewed in Peisajovich \& Shai, 2002, 2003; Morrison, 2003). In particular, the HR domains (HR1 and HR2 or HR-A and HR-B) have been shown to possess unique structures in different conformational states of the F protein (Lamb, 1993; Lamb et al., 1999; Peisajovich \& Shai, 2002, 2003; Morrison, 2003). It has also been shown that both HR1 and HR2 or HR2 alone have fusion-inhibition effects on virus entry (Lamb et al., 1999; Peisajovich \& Shai, 2002, 2003; Morrison, 2003). In the case of $\mathrm{SeV}$, only HR2 shows an inhibitory effect (Rapaport et al., 1995). In the current model, at least three conformational 
states of the $\mathrm{F}$ protein have been proposed: pre-fusion native conformation, pre-hairpin intermediate conformation and post-fusion coiled-coil conformation (Lamb et al., 1999; Peisajovich \& Shai, 2002, 2003; Morrison, 2003). HR1 and HR2 are believed to be separated in the pre-fusion native state and the intermediate state, but may be in different conformations. However, it is clear that they interact with each other to form a six-helix coiled-coil bundle in the postfusion state as shown by biochemical investigation of a number of paramyxoviruses (Lamb et al., 1999; Peisajovich \& Shai, 2002, 2003; Morrison, 2003) and the crystal structures of human respiratory syncytial virus (hRSV) and simian parainfluenza virus 5 (SV5) (Baker et al., 1999; Zhao et al., 2000), both of which are members of the Paramyxoviridae. In this six-helix coiled-coil bundle, both HR1 and HR2 are $\alpha$-helical and three of the HR1 domains interact with each other to form a homotrimeric coiledcoil core surrounded by three HR2 domains in an antiparallel manner (Lamb et al., 1999; Eckert \& Kim, 2001). This six-helix coiled coil bundle has been called the fusion core and is believed to be the most stable structure of the $F$ protein. The energy release during the conformational state transitions is believed to be used for viral-cellular membrane fusion.

In an attempt to understand the structural basis and element requirements of the sixhelix bundle formation of Paramyxoviridae family members, we have carried out a number of paramyxovirus fusion-core analyses by methods including biochemistry, biophysics and X-ray crystallography (e.g. Zhu et al., 2002; Yu et al., 2002). Although the SeV HR1 and HR2 regions have previously been studied by Shai and colleagues (Ghosh et al., 1997, 1998), they did not show the formation of the six-helix bundle complex because they used shorter amino-acid sequences for these experiments which did not cover the whole HR regions (see Fig. 1 for details). In their experiments, they only showed that the two regions interact with each other with $\alpha$-helix structure by labelling the HR2 with fluorescent reagents. Therefore, in this study we have designed a single-chain HR1-linker-HR2 of $\mathrm{SeV}$ (2-Helix) and expressed the protein in a prokaryotic system. Subsequently, a series of biochemical, biophysical and X-ray diffraction techniques were employed for analysis. We show here that HR1 and HR2 of SeV F protein interact with each other and form a typical six-helix coiled-coil bundle: the fusion core. This stable protein has been crystallized and the detailed crystal structure has been solved, showing a typical six-helix coiled-coil structure, which will be reported elsewhere.

\section{Materials and methods}

\subsection{Gene construction, protein expression} and purification

The F-protein sequence used in this study was derived from $\mathrm{SeV}$ strain T5 (GenBank Accession No. M69046) and its functional/ structural modules/domains are depicted in Fig. 1(a). To choose the HR1 and HR2 sequences the LearnCoil-VMF program (Singh et al., 1999) was used, as it was specifically designed for identification of viral heptad-repeat coiled coils and has been successfully used for a number of virusfusion proteins. These regions have been studied previously by Shai and coworkers and the peptides were named SV150 or SV163 for HR1 and SV465 or SV473 for HR2 (Rapaport \& Shai, 1994; Rapaport et al., 1995; Ghosh et al., 1998). As can be seen in Fig. 1(b), the sequences we chose were closer to the prediction than those used in the series of studies carried out by Shai and coworkers. For the 2-Helix gene construction, the HR1 region used was derived from amino acids $142-188$ and the HR2 region used was derived from amino acids 462-493. The HR1 region is quite close to the prediction, but for the sake of solubility eight amino acids were omitted. The HR2

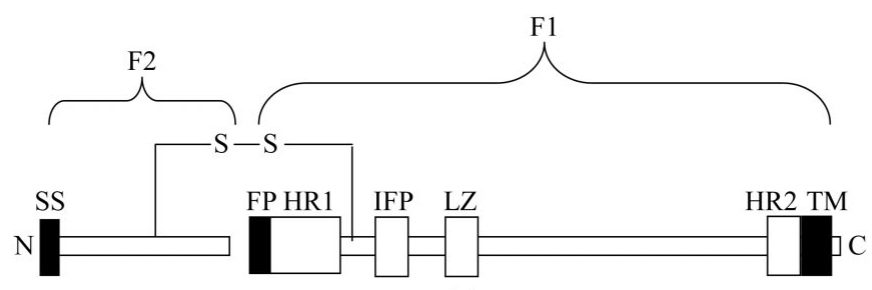

(a)

Predicted HR1: AGIALAEAREAKRDIALIKESMTKTHKSIELLQNAVGEQILALKTLQDFVNDEIK

$\begin{array}{lr}\text { Shai SV150: } & \text { DIALIKESMTKTHKSVELLQNAVGEQILALKTLQDFV } \\ \text { Shai SV163: } & \text { KSVELLQNAVGEQILALKTLQDFV } \\ \text { Our construct: } & \text { AEAREAKRDIALIKESMTKTHKSIELLQNAVGEQILALKTLQDFVND } \\ \text { Predicted HR2: } & \text { DISLNLADATNFLQDSKAELEKARKILSEVGR } \\ \text { Shai SV465: } & \text { LNLADATNFLQDSKAELEKARKILSEVGRWY } \\ \text { Shai SV473: } & \text { FLQDSKAELEKARKILSEVGRWY } \\ \text { Our construct: } & \text { DISLNLADATNFLQDSKAELEKARKILSEVGR }\end{array}$

2-Helix: HR1 SGGRGG HR2

*There is a conserved substitution from valine to isoleucine at amino acid 165 in HR1 between Shai's and our strains. (b)

Figure 1

Location of HR regions used in this study in the F-protein gene and their amino-acid sequences. (a) Schematic diagram of SeV F protein showing F1 and F2 and the functional/structural modules (domains) in addition to HR1 and HR2. SS, signal sequence; FP, fusion peptide; IFP, internal fusion peptide; LZ, leucine-zipper domain (amino acids 201-229); TM, transmembrane region. 'S-S' represents the disulfide bond linking F1 and F2. (b) Sequence alignment of HR1 and HR2 predicted by LearnCoil-VMF (Singh et al., 1999) or used by Shai and coworkers and here (our construct is named 2-Helix, in which HR1 and HR2 are linked by a linker, SGGRGG.) 
dichroism (CD) spectroscopy as described previously (Zhu et al., 2002).

\subsection{Crystallization, X-ray data collection and processing}

The 2-Helix protein was buffer-exchanged into $20 \mathrm{~m} M$ Tris- $\mathrm{HCl}$ buffer $\mathrm{pH} 8.0$ and concentrated to $10 \mathrm{mg} \mathrm{ml}^{-1}$. The initial crystallization conditions were screened using the hanging-drop vapour-diffusion method with sparse-matrix crystallization kits (Crystal Screens I and II; Hampton Research, Riverside, CA, USA). $1 \mu$ l protein solution was mixed with $1 \mu \mathrm{l}$ reservoir solution and equilibrated against $200 \mu \mathrm{l}$ reservoir solution. The crystallization trays were kept at 291 K. 2-Helix protein crystals were obtained and the conditions were optimized to obtain better diffracting crystals.

The X-ray diffraction data for a single crystal were collected at $100 \mathrm{~K}$ with a MAR Research (Norderstadt, Germany) imageplate detector using $\mathrm{Cu} K \alpha$ radiation from a Rigaku rotating-anode generator. The collected data were processed using the $H K L$ programs DENZO and SCALEPACK (Otwinowski \& Minor, 1997).

\section{Results and discussion}

As described in $\S 2$, the SeV HR1 and HR2 regions were predicted using the LearnCoil$V M F$ program (Singh et al., 1999) (Fig. 1) and our constructed HR1 and HR2 regions were subsequently amplified by PCR and cloned into the GST-fusion expression vector pGEX-6P-1. To facilitate crystallization, the two regions were linked by a flexible six-amino-acid linker and the construct (HR1-linker-HR2) was named 2-Helix. This strategy has also been used in our laboratory for other paramyxoviruses (e.g. Zhu et al., 2002). Because the LearnCoil-VMF prediction gave longer aminoacid sequences for both regions than those used by Shai and coworkers, we chose the HR1 region (47 amino acids) to be close to the prediction and the HR2 region (32

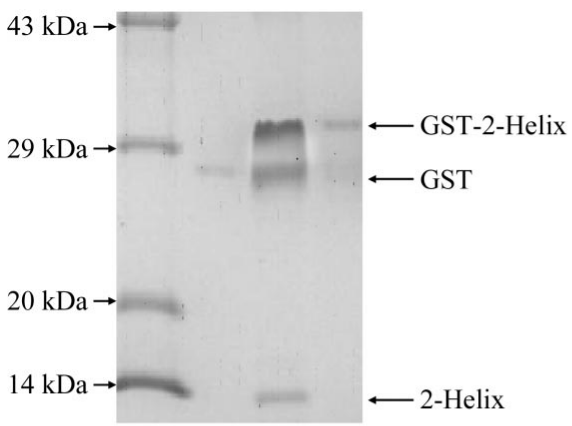

(a)

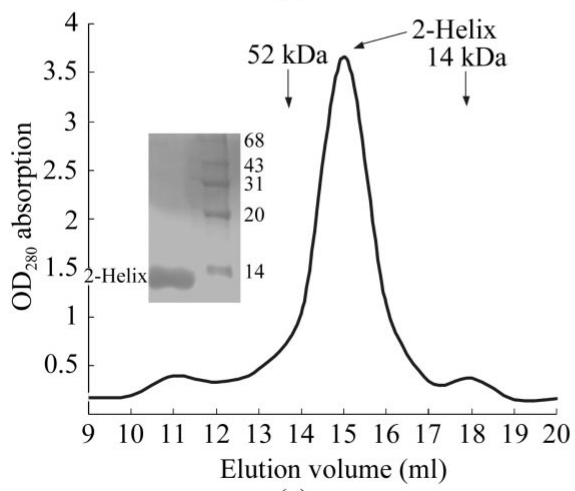

(c)

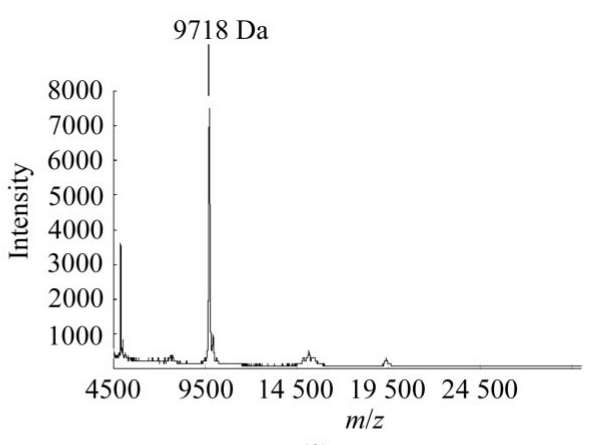

(b)

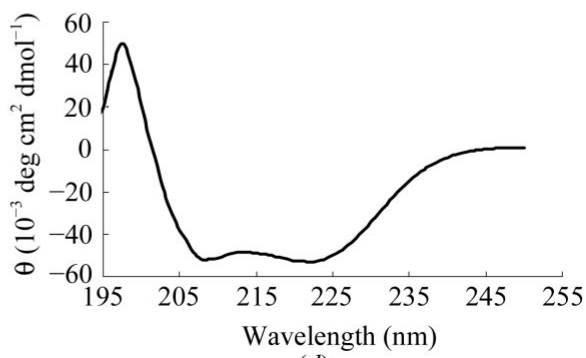

(d)

Figure 2

Characterization of the 2-Helix protein. (a) SDS-PAGE analysis of the proteins. 2-Helix protein was expressed as a GST-fusion protein and cleaved by GST-3C enzyme. Lane $M$, protein molecular-weight markers in $\mathrm{kDa}$ as indicated; lane 2, purified GST control protein; lane 3, 2-Helix protein after GST-3C cleavage; lane 4, purified GST-fusion 2-Helix. (b) MALDI-TOF mass-spectrometry profile of the 2-Helix. It is clearly shown that the molecular weight of the major peak is $9.718 \mathrm{kDa}$, matching the molecular weight of the 2-Helix monomer. $(c)$ Gel-filtration analysis of the 2-Helix protein. In the Superdex G75 column elution pattern, a clear peak between the eluted volumes corresponding to 52 and $14.4 \mathrm{kDa}$ standards indicates the 2-Helix protein. It is of about $30 \mathrm{kDa}$ in weight, indicating the formation of a 2-Helix trimer or six-helix bundle. The inset picture is Tris-tricine SDS-PAGE analysis of the peak, indicating the existence of only 2-Helix protein in the peak. The molecular standards run are indicated in $\mathrm{kDa}$. (d) $\mathrm{CD}$ spectra of the 2-Helix protein. A typical $\alpha$-helix secondary structure with double minima at 208 and $222 \mathrm{~nm}$ was seen for the 2-Helix protein sample.

Table 1

Diffraction data statistics.

\begin{tabular}{ll}
\hline Space group & $R 3$ \\
Unit-cell parameters $\left(\AA,{ }^{\circ}\right)$ & $a=42.149, b=42.149$, \\
& $c=182.577, \alpha=90$, \\
& $\beta=90, \gamma=120$ \\
Wavelength $(\AA)$ & 1.5418 \\
Resolution $(\AA)$ & 2.5 \\
Observed reflections & 24732 \\
Unique reflections & 5274 \\
Completeness $(\%)$ & $100(100)$ \\
$I / \sigma(I)$ & $5.5(5.2)$ \\
$R_{\text {sym }}(\%)$ & $12.9(43.6)$ \\
\hline
\end{tabular}

amino acids) to be identical to the prediction as shown in Fig. 1. Five amino acids from the $\mathrm{N}$-terminus and three from the $\mathrm{C}$-terminus in the HR1 region were omitted compared with the prediction in order to increase the solubility of the polypeptide.

When 2-Helix (85 amino acids in total) was expressed in Escherichia coli as a GSTfusion protein, it was soluble and could subsequently be cleaved from the fusion partner GST (Fig. 2a). The protein was purified by affinity column and gel filtration with high homogeneity as shown by MALDI-TOF (Bruker Daltonics Biflex III) mass spectrometry (Fig. $2 b)(9.718 \mathrm{kDa}$, the molecular weight of 85 amino acids in 2-Helix protein). A single symmetrical peak of 2-Helix protein was observed on gel filtration (Fig. 2c) and estimation of the molecular weight showed the formation of the trimer (approximately $30 \mathrm{kDa}$ ), $3 \times$ HR1-HR2, consistent with other paramyxovirus fusion cores, including those of hRSV and SV5, the crystal structures of which have been solved (Baker et al., 1999; Zhao et al., 2000).

To test the secondary structure of the purified 2-Helix protein, CD spectrometry was carried out and the results show that 2-Helix protein forms a typical $\alpha$-helix structure (Fig. $2 d$ ). The melting temperature of the 2-Helix protein is as high as $370 \mathrm{~K}$ (data not shown), indicating that it is extremely stable, and the virus fusion core is characterized by the coiled-coil structure (Lamb et al., 1999; Eckert \& Kim, 2001). All these results including the gel-filtration data support the formation of a stable coiled-coil six-helix bundle in the 2-Helix protein.

Crystallization of 2-Helix protein proved to be easy under several conditions (data not shown), indicating its stable tightly packed structure. A crystal grown (within days) from a condition consisting of $0.1 \mathrm{M}$ citric acid pH 2.5, 20\% PEG 4000 (Fig. 3) diffracted X-rays to $2.5 \AA$ and belonged to space group $R 3$, with unit-cell parameters $a=b=42.149, c=182.57 \AA$ A Data-collection statistics are given in Table 1. Molecularreplacement calculations were performed 


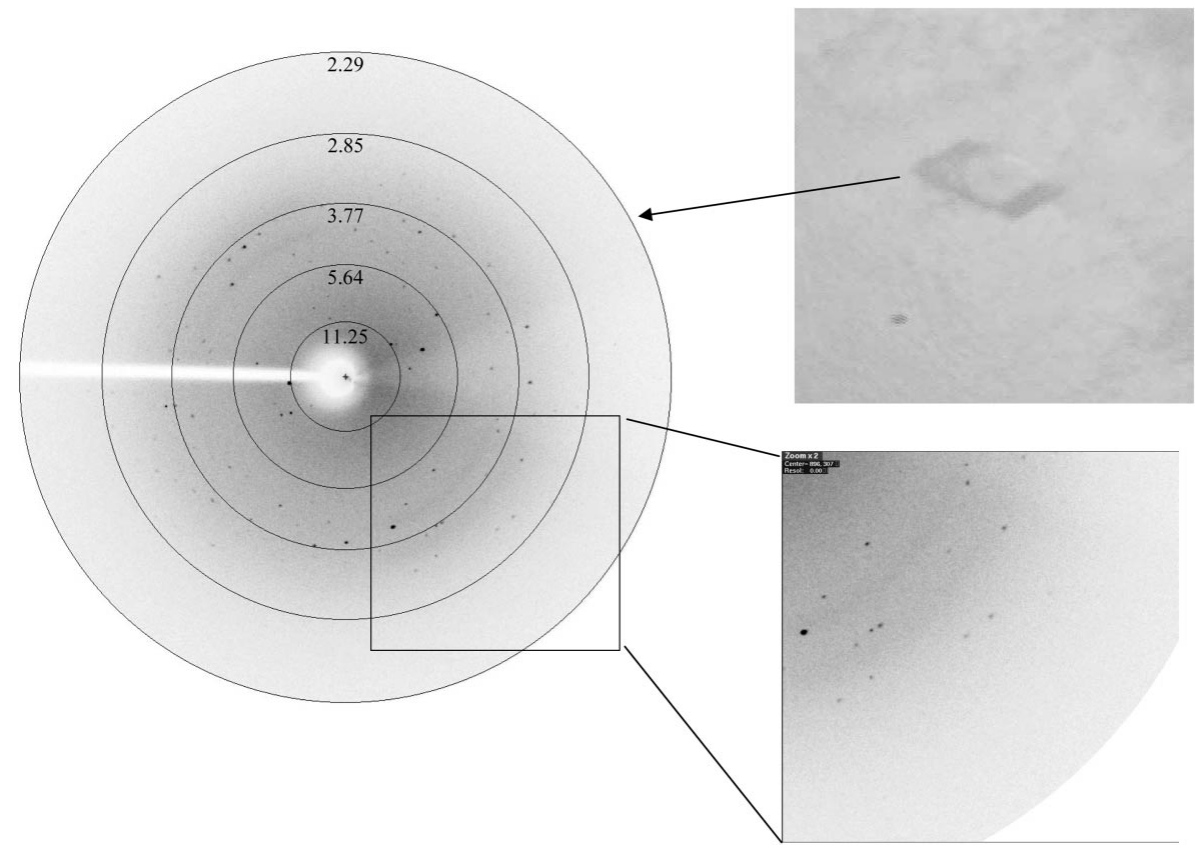

Figure 3

Sample 2-Helix crystal and its X-ray diffraction pattern.

using the program CNS (Brünger et al., 1998) with the crystal structure of SV5 N1/C1 trimer as a search model (PDB code 1svf; Baker et al., 1999). The structure has been solved, showing a typical six-helix coiled-coil structure, which will be reported elsewhere. Recently, a cryo-electron microscopic (EM) structure of the $\mathrm{SeV} F$ protein was solved at $16 \AA$ and a six-helix bundle structure was proposed in the stalk region (Ludwig et al., 2003), which must be formed of HR1 and HR2 regions. Using the information from the HR1-HR2 fusion-core structure, we should be able to produce a

Baker, K. A., Dutch, R. E., Lamb, R. A. \&
Chang, P.-C., Hsieh, M.-L., Shien, J.-H., Graham, D. A., Lee, M.-S. \& Shieh, H. K. (2001). J. Gen. Virol. 82, 2157-2168.

Eckert, D. M. \& Kim, P. S. (2001). Annu. Rev. Biochem. 70, 777-810.

Ghosh, J. K., Ovadia, M. \& Shai, Y. (1997). Biochemistry, 36, 15451-15462.

Ghosh, J. K., Peisajovich, S. G., Ovadia, M. \& Shai, Y. (1998). J. Biol. Chem. 273, 27182-27190.

Lamb, R. A. (1993). Virology, 197, 1-11.

Lamb, R. A., Collins, P. L., Kolakofsky, D., Melero, J. A., Nagai, Y., Oldstone, M. B. A., Pringle, C. R. \& Rima, B. K. (2000). Virus Taxonomy: Classicification and Nomenclature of Viruses, 7th Report, edited by M. H. V. van Regenmortel, C. M. Fauquet, D. H. L. Bishop, E. B. Carstens, M. K. Estes, S. M. Lemon, J. Maniloff, M. A. Mayo, D. J. McGeoch, C. R. Pringle \& R. B. Wickner, pp. 549-561. San Diego: Academic Press.

Lamb, R. A., Joshi, S. B. \& Dutch, R. E. (1999). Mol. Membr. Biol. 16, 11-19.

Ludwig, K., Baljinnyam, B., Herrmann, A. \& Bottcher, C. (2003). EMBO J. 22, 3761-3771.

Morrison, T. G. (2003). Biochim. Biophys. Acta, 1614, 73-84.

Otwinowski, Z. \& Minor, W. (1997). Methods Enzymol. 276, 307-326.

Peisajovich, S. G. \& Shai, Y. (2002). Trends Biochem. Sci. 27, 183-190.

Peisajovich, S. G. \& Shai, Y. (2003). Biochim. Biophys. Acta, 1614, 122-129.

Rapaport, D., Ovadia, M. \& Shai, Y. (1995). EMBO J. 14, 5524-5531.

Rapaport, D. \& Shai, Y. (1994). J. Biol. Chem. 269, 15124-15131.
$\mathrm{F}$ protein in virus membrane fusion.

This work was supported by a grant from The National Natural Sciences Foundation of China (NSFC) (Grant No. 30228025).

\section{References} Jardetzky, T. S. (1999). Mol. Cell, 3, 309-319.

Brünger, A. T., Adams, P. D., Clore, G. M., DeLano, W. L., Gros, P., Grosse-Kunstleve, R. W., Jiang, J.-S., Kuszewski, J., Nilges, M., Pannu, N. S., Read, R. J., Rice, L. M., Simonson, T. \& Warren, G. L. (1998). Acta Cryst. D54, 905 921.
Singh, M., Berger, B. \& Kim, P. S. (1999). J. Mol. Biol. 290, 1031-1041.

Wang, L., Harcourt, B. H., Yu, M., Tamin, A., Rota, P. A., Bellini, W. J. \& Eaton, B. T. (2001). Microbes Infect. 3, 279-287.

Yu, M., Wang, E., Liu, Y., Cao, D., Jin, N., Zhang, C. W.-H., Bartlam, M., Rao, Z., Tien, P. \& Gao, G. F. (2002). J. Gen. Virol. 83, 623-629.

Zhao, X., Singh, M., Malashkevich, V. N. \& Kim, P. S. (2000). Proc. Natl Acad. Sci. USA, 97, 14172-14177.

Zhu, J., Zhang, C. W.-H., Qi, Y., Tien, P. \& Gao, G. F. (2002). Biochem. Biophys. Res. Commun. 299, 897-902. 